\title{
Study on Railway Signal Computer Interlocking System
}

\author{
Xiaohui Xu \\ Weifang University of Science \& Technology, Shandong, Shouguang, 262700, China.
}

Keywords: Railway signal; Computer interlocking; Trouble discussion; Principle.

\begin{abstract}
This paper mainly discussed the railway signal computer interlocking system, including the bas is of computer interlocking system, computer interlocking principle, fault of computer interlocking system maintenance. The study is focused on the working principle of the computer interlocking, including its hardware structure, working principle and the reliability and safety design.
\end{abstract}

\section{Introduction}

At present, the computer interlocking control system is in practical stage, with the accumulation of practical experience, the performance of the system is also improving. The computer interlocking control system of our country mainly adopts computer interlocking control system which is composed of general industrial control computer. In recent years, the system of double 2-vote-2 redundancy is introduced by two CPU constituting a subsystem executes the interlock tasks (host), two different CPU in hot standby (machine), which greatly improves the computer interlocking control system reliability and security, and the maintenance is convenient. The technological transformation of the main trunk are considered as the two by two out of two systems. At present, the computer interlocking control system has been equipped with thousands of stations.

\section{Computer interlocking systembase}

Computer interlocking overview. In order to ensure the traffic safety and the necessary carrying capacity, signal, switch and route must be necessary technical means to keep certain constraints and operation sequence, the control and operation sequence that interlock, using computer technology to realize the system to become a computer in terlocking system.

\section{Computer interlocking main technical conditions.}

1) Computer interlocking can meet the needs of various railway station (field) of scale and transportation operation, ensuring the driving safety and improving transportation efficiency which have great information quantity and networking capability.

2) The structure of computer interlocking system is hardware redundancy, such as the double hot standby, two take three or two by two. Reliability index: average failure interval (MTBF) is greater than or equal to 106h; safety index: the average risk of the output interval is greater than or equal to 1011h.

3) Use of computer interlocking involves the safety of the circuit fault - Safety Principle; the circuit fault can be found immediately. When the fault can endanger the driving safety and can cut off the dangerous side of the systemoutput.

4) The structure of computer hardware is a hierarchy, such as human and dialogue layer, interlocking application layer and execution layer.

5) The computer interlocking has the communication ability through the communication front processor and the communication net and other system, and the data communication of the dispatching command systemaccords with the relevant regulations.

6) The software system of computer interlocking achieves the reliability and security of the software testing system, and all the programs are modularized, structured and standardized. 
7) The interface and channel of computer interlocking can guarantee the high stability and high reliability for long term use.

8) The computer interlocking can through the external data channel or computer network and other automation or management system.

9) The computer interlocking is equipped with two independent power supplies and has an automatic transfer function to en sure uninterrupted power supply.

10) The computer interlocking takes the necessary anti electromagnetic interference and lightning protection measures, in order to assure that the equipment can work in the use of the harsh levels of the environment, .

11) The grounding resistance of the signal equipment is nothing more than 10 , and the grounding device of the ground wire for the protection of the safe ground of the electronic equipment is no more than 4. For the inten se lightning area, ground setting has also taken special measures.

12) As the basic part of the system, the monitoring subsystem provides the function of monitoring, alarming, statistic, analy sis, management, remote diagnos is and maintenance for the maintenance and use of the Department.

13) According to the need to establish the emergency disk, when the computer interlocks failure control switch and guide signal.

\section{Computer interlocking principle}

Hardware component of Computer Interlocking System. The computer interlocking system is the industrial control computer system which is used to realize the monitoring and control of the railway station transportation production process. It is composed of industrial con trol computer and the production process of two parts composition and industrial control computer which refers to the characteristics and requirements according to the production process control and design of computer. It includes two parts: hardware and software. In the field of railway signal, the production process refers to the industrial control computer through the process input output channel and relay combined circuit of monitoring objects such as switch and signal and for real-time monitoring. Typical computer interlocking system hard ware is given in Fig. 1.

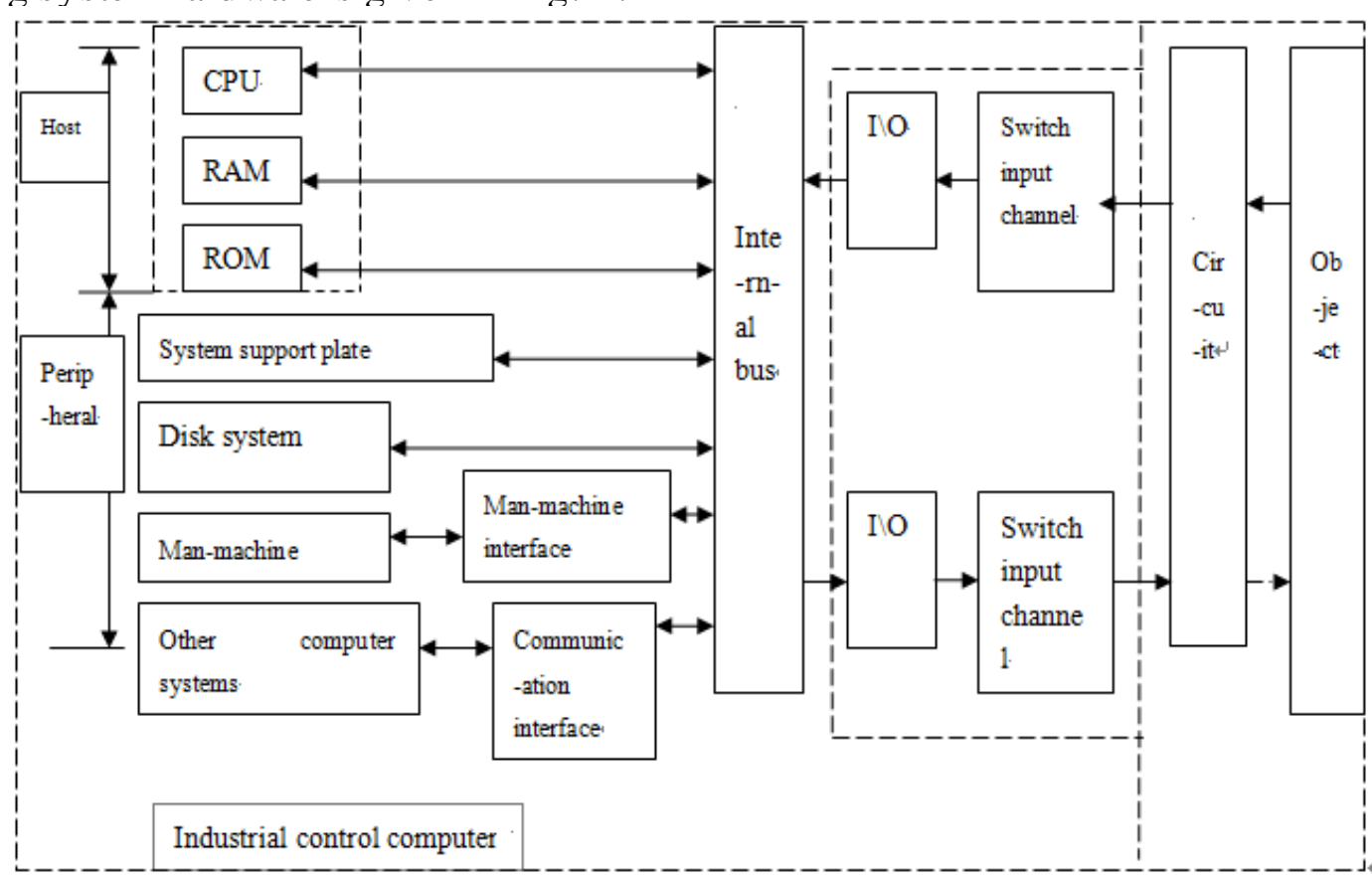

Fig. 1 Hardware component diagram of Computer Interlocking System

The computer interlocking system of comprehensive performance far exceeds the relay interlocking system, so the interlocking system of the station by the relay device to the computer interlocking 
system is transformed has grown up to be an irreversible trend. In particular, the advantages of computer interlocking system are in the aspects of timeliness, safety, reliability, maintainability and price ratio.

Computer interlocking system is using the existing industrial control computer, develop ing a set of dedicated hardware and software system to achieve signal, route and switch between the interlocking relationship, so it essentially is a meet the fault - Safety Principle of signal interlock logic system, the role of computer in the system is operational command and a variety of field to input representation of information to be read, according to the computer's internal condition of logic operation, determine the output control information to the actuators to achieve digital input of multi variable and multi variable digital output such a complex transfer function transformation. Fig. 2 is a logic systemschematic.

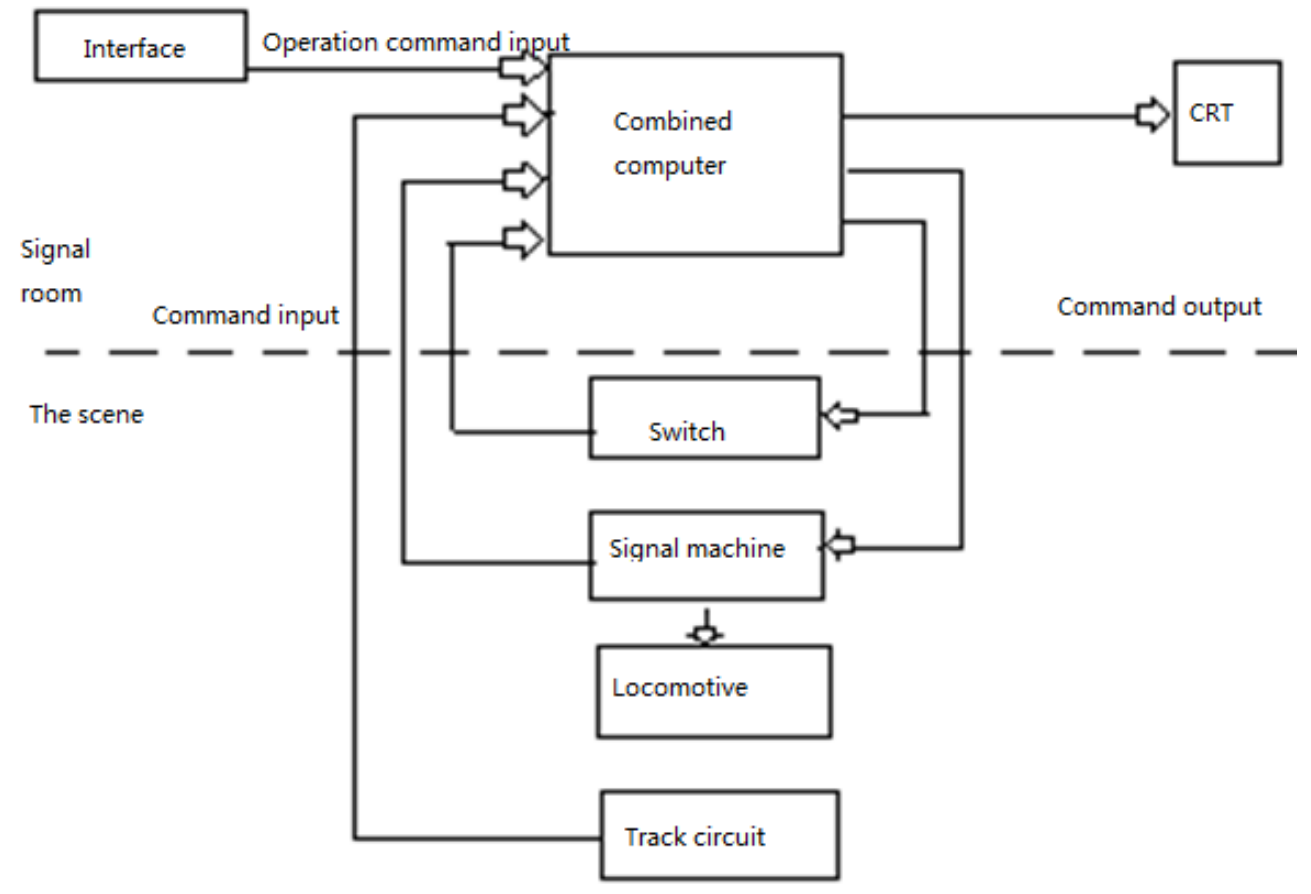

Fig. 2 Principle diagram of interlocking logic operation system

Basic principle of Computer Interlocking System. Fro m the architecture of the computer system, it belongs to the two levels distributed control system, which breaks the old centralized signal system mode and has the features of modularization and hierarchy.Modular refers to interlock machine main module, PLC and signal binding module, the hierarchy refers to the system of representation layer, the interlocking computing layer, check the driver layer, combined with the circuit layer and monitoring object layer and other five physical levels. This structure has the advantages of the station scale size, different job requirements, which does not alter the interlocking software based by modified station static data and the addition of the corresponding hardware module to meet the requirements of the expansion of the system, combined with the adv anced control system design process makes the system debugging period and the construction site, open cycle are shortened, with good economic and practical.

1) Man machine dialogue layer

Coming from the keyboard, mouse and other operational input, the serial port is served by interlocking computer, while the display station on the graphics displays information. The field scale larger resulting in computer interlocking burden is heavier or needs multiple terminal operation, operational command acquisition machine set of operational command input of discriminant validity and converted in to a predetermined transmission format to the computer interlocking.

2) Interlocking computing layer

Computer interlocking system is the core part of the system, bear the input operation of the discriminant, interlock signal conditioning and analysis, logic operation and control command 
generation, fault diagnosis task, the reliability and security of the system in the overall failure, safety performance has greater impact, hj04a system sets two microcomputer interlocking, one of them is cold standby machine, manual switch.

3) Review driver layer

Checking drive layer is composed of PLC, and its commitment to the acquisition information and operation commands are issued by the microcomputer interlocking transformation tasks for the fail safe control signal, as one of the important link of the security of the sy stem design, PLC also bears interlocking microcomputer formed operation command, check the barrier function of.

4) Circuit layer

One of the tasks of the circuit is to realize the security logic conversion of the drive signal and the PLC output signal, so that the input and output information of the PLC can have the fault safety performance. The two tasks are to use a special circuit specification monitoring and control process, which includes the information collection mechanism and device driver process.

5) Monitor object layer

The monitoring equipment is the equipment of the interlocking system, that is, the switch, the signal machine and the track circuit.

\section{Trouble maintenance and discussion of Computer Interlocking System}

\section{Computer unit fault}

1) Interlocking machine

STD board fault, the concrete performance in: STD layer running lights stop flashing, receiving lights, internupt lamp, the collection layer, driver layer indicator light stop flash, failure is CPU board fault,

Processing method: replace the CPU board; STD layer interrupt 2 lights, running lights, but sending and receiving light flashing lights, according to the off position, replacing the STD-01 (and monitoring machine communication and in terlocking machine communication).

BJ-A0 board fault, STD layer running lights, interrupt lights, alarm lights are not flashing, the collective layer is normal.

Processing method: replace BJ plate or fastening socket.

2) Monitor machine

PC-01 network card failure and its interlocking machine STD layer first assembles light is not flashing and other lights are normal, and "interlocking machine communication interrupt" tips; Ethernet failure prompts machine monitoring and maintenance of computer communication interrupt, VGA cards have fault, screen does not display and graphics have defects.

Processing method: replace the PC-01 card and retum to normal, which is required to replace the card or plug is not strong.

Communication line fault. Bus plugs loose or bad plug, interlock machine cannot monitor and communication. LS is loose plug or socket, working machine interlocking machine synchronous machine cannot be prepared and,

Methods: check if the plug is loose and only fully in contact with the online button.

Switching fault. When the zero level switch board of the interlocking machine breaks off, the error of the switch is controlled and the communication between the monitoring computer and the interlocking machine is interrupted. To eliminate the trouble and monitor con sole and digitizer switch, board failu re will lead to consoling display and digitizer cannot normally be with the monitor switched to the work of the monitoring computer, cau sing the dis play no display.

Processing methods: this time to exclude the influence of outward power factor, 1 need s to replace the switch board, 2 need s to change the switch board, troubleshooting. 3.4 power failures

The failure of the dynamic regulated power supply can cause the failure of all the dynamic relay and cannot drive the outdoor equipment. 
The load of UPS power supply, STD power supply, power supply, power supply, power supply and monitoring power supply are all failure, and the load of the belt cannot be turned on.

Processing method: check the input power supply works, output power works if they are all normal, which needs to change the power supply board parts according to the fault point, recovery failure phenomenon.

\section{Conclusions}

This paper mainly describes the hardware structure of the computer interlocking system, equipment selection and power allocation, and other principles and methods. The two by two system structures of the computer interlocking control system, especially the research for reliability and fault tolerance techniques, computer interlocking technology has matured, vigorously promote the use. According to computer interlocking application and research of the country, because the computer has a strong function in logic function and information processing, the signal, switch control circuit and a combination of intemal wiring and the signal machine, switch, track circuit, part of the equipment state information acquisition circuit and interlock machine interface circuit and control.

\section{References}

[1] Hongze $\mathrm{Xu}$. The principle and application of computer chain control system. Beijing: China Railway Press.2008.

[2] Zhixi Zhao. "Computer chain control technology". Beijing: China Railway Press.1999.

[3] Hongze Xu. The principle and application of computer interlocking control system for railway station signal. Beijing: China Railway Press.2006.

[4] Yongxin Wang. "Automatic control of station signal". Beijing: China Railway Press.2010.

[5] Wenqing He. "6502 electrical centralized circuit". Beijing: China Railway Press.2007. 